\title{
Compatibilidad y eficiencia in vitro de Glomus intraradices en coníferas nativas del sur de Chile
}

\author{
Compatibility and efficiency in vitro of Glomus intraradices
} on native conifers from Southern Chile

C.D.O.: 181.351

\author{
ROBERTO GODOY ${ }^{1}$, RUBEN CARRILLO ${ }^{1}$ y HERNAN PEREDO ${ }^{2}$ \\ ${ }^{1}$ Instituto de Botánica, ${ }^{2}$ Instituto de Silvicultura, Universidad Austral de Chile, Casilla 567, Valdivia, Chile.
}

\section{SUMMARY}

The forest communities in southern Chile present a mycorrhizal vesicular-arbuscular dominance which has been documented in most wood plants of silvicultural interest, particularly in the endemic conifers.

This research listed the compatibility and efficiency of vesicular-arbuscular mycorrhiza in four conifer species, representative of native forest communities.

A controlled inoculation greenhouse essay with Glomus intraradices was performed in: Fitzroya cupressoides, Podocarpus nubigena, Podocarpus saligna and Saxe-gothaea conspicua. At the end of the essay (20 weeks), the mycorrhizal colonization degree and morphometric parameters were measured by means of the quality index of seedlings to determine the efficiency of simbiont fungi.

The results presented here confirm a high compatibility and a strong mycorrhizal colonization degree with the endosimbiont. The values show a specificity in the four conifers species and great mycorrhizal dependency.

The statistical analyses of the morphometric variables indicate that these were significantly higher than the control. The results show that the morphometric variables of the stem are good indicators of the quality of seedlings. The quality index of each conifer species was higher in the treatment than in the control plants.

This data allows us to propose plant mycorrhization as a good alternative to future forestation programs.

\section{RESUMEN}

En la mayoría de las plantas leñosas de importancia silvicultural, presentes en las comunidades boscosas del sur de Chile, se ha documentado la dominancia del tipo micorrícico vesículo-arbuscular, particularmente en las coniferas endémicas.

El presente estudio consistió en un ensayo de inoculación micorrícica con Glomus intraradices bajo condiciones de invernadero, para lo cual se utilizaron plántulas de cuatro especies de coniferas de las comunidades boscosas nativas: Fitzroya cupressoides, Podocarpus nubigena, Podocarpus saligna y Saxe-gothaea conspicua, con el objeto de determinar la compatibilidad y eficiencia del simbionte.

Al término del ensayo (20 semanas) se determinó el grado de colonización micorrícica y se midieron variables morfométricas para establecer la eficiencia del endosimbionte, mediante el índice de calidad de las plántulas.

Los resultados confirmaron la compatibilidad del endosimbionte, expresado en un alto grado de colonización y especificidad en cada una de las especies, lo cual indica una gran dependencia micorrícica.

Las variables morfométricas analizadas en los tratamientos fueron, en general, significativamente mayores que el control, destacando como buenos indicadores aquellas derivadas del tallo. El índice de calidad obenido fue estadísticamente significativo para cada especie huésped en relación al control. Los resultados permiten proyectar la alternativa de micorrización como una herramienta eficiente para futuros programas de reforestación.

\section{INTRODUCCION}

El sur de Chile, caracterizado por poseer la masa boscosa más austral del planeta y que corresponde al remanente de una flora cuya extensión alcanzó históricamente latitudes aún más meridionales que las actuales, no se encuentra ajeno al impacto antrópico, donde el recurso forestal nativo constituye hoy en día la principal área de explotación. Si consideramos que la flora autóctona del sur de Chile posee alrededor de 84 taxa de representantes arbóreos, no es menos cierto que un $50 \%$ de 1 os 
mismos son endémicos, con un gran significado genético fitogeográfico y ecológico (Armesto et al., 1992), varios de los cuales, particularmente las especies de coniferas, se encuentran con serios problemas de conservación (Godoy y Mayr, 1989; Armesto 1992; Parker y Donoso, 1993; Godoy et al., 1994).

Sobre el bosque nativo chileno existen mitos atribuidos al desconocimiento y a la carencia de estudios, por lo que algunas especies endémicas no están adecuadamente protegidas y gran parte de las comunidades se encuentran degradadas o han sido reemplazadas por monocultivos de especies introducidas (Armesto, 1992; Donoso, 1993; Godoy et al., 1994; Lara y Veblen, 1993). Situaciones semejantes se solucionan a nivel mundial, planteando una estrategia de desarrollo económico viable que respete la integridad del ambiente (Mc Neill, 1989). Lo anterior es posible de realizar mediante la restauración ecológica, que incorpore la investigación básica y aplicada al manejo de los recursos naturales renovables (Danielson 1988; Alien et al., 1989).

Uno de los aspectos de gran relevancia enfatizados en la última década es considerar en las prácticas de manejo silvicultural y de restauración ecológica de áreas devastadas el estudio de la rizósfera y la aplicación de hongos micorrícicos (Perry et al., 1987; Danielson, 1988; Carrillo et al., 1992; Godoy et al., 1994).

La estructura y estabilidad de una asociación vegetal, al igual que del ecosistema en general, se encuentran fuertemente influenciadas por la simbiosis micorrícica (Grimme et al., 1989; Read, 1991; Godoy et al., 1994). Esta simbiosis mutualística conlleva una serie de ventajas para el huésped, principalmente en lo que se refiere a la absorción y transporte de nutrientes y agua, a través de una estrecha red de hifas en el suelo, permitiendo el traspaso de sustancias tanto a nivel intra e interespecífico (Harley, 1989).

Estudios realizados por Godoy y Mayr (1989), sobre la caracterización micorrícica en nueve especies de coniferas nativas de Chile, señalan el alto grado de colonización simbiótica que presentan los hospederos. Posteriormente, HildebrandVogel et al. (1992) y Godoy et al. (1994) documentan la importancia de las micorrizas en comunidades de coniferas nativas, con énfasis en los bosques de Fitzroya cupressoides en el sur de Chile.

El objetivo general del presente trabajo es con- tribuir al estudio de 4 especies de coniferas nativas asociadas a los bosques siempreverdes de la Cordillera de la Costa, del sur de Chile, en cuya primera fase se pretende determinar mediante bioensayos de inoculación controlada la compatibilidad y eficiencia del simbionte Glomus intraradices Schenck \& Smith.

\section{MATERIAL Y METODOS}

Para el desarrollo del bioensayo fue necesario obtener plántulas vía agámica, mediante la producción de esquejes enraizados de; Fitzroya cupressoides (Mol.) Johnst, Podocarpus nubigena Lindl., Podocarpus saligna D. Don y Saxe-gothaea conspicua Lindl. El material fue obtenido del bosque original ubicado en la Cordillera de la Costa, Valdivia $\left(40^{\circ} 13^{\prime} \mathrm{S}-73^{\circ} 30^{\prime} \mathrm{W}\right)$. Para cada especie de conifera fue seleccionado un individuo adulto sano, cuyas estacas obtenidas de tamaño uniforme (10 cm de long. y $0.3 \mathrm{~cm}$ de diámetro basal) fueron desinfectadas con alcohol al $50 \%$ en su porción basal y posteriormente sumergidas en una solución hidroalcohólica de ácido indol-butírico (AIB) de $2.000 \mathrm{ppm}$, por un período de 5 segundos (Hartmann y Kester, 1964). Posteriormente fueron plantadas en una cama de propagación estéril, compuesta de turba-arena $(1: 1 \mathrm{v} / \mathrm{v})$ y de profundidad $10 \mathrm{~cm}$. El sustrato fue mantenido a una temperatura de $22^{\circ} \mathrm{C}$; la humedad del medio aéreo se mantuvo mediante aspersión de agua en forma intermitente, con el suelo a capacidad de campo.

Después de 5 meses se seleccionaron plántulas enraizadas de tamaño uniforme: $F$. cupressoides $15 \mathrm{~cm}, P$. nubigena $12 \mathrm{~cm}, P$. saligna $12 \mathrm{~cm} \mathrm{y} S$. conspicua $15 \mathrm{~cm}$, para ser trasladadas a maceteros de 11 de capacidad. El sustrato utilizado corresponde al suelo del bosque original, de textura franco-arenosa con $\mathrm{pH} 4.6$, carbono total: $4.1 \%$, nitrógeno total: $0.15 \%, \mathrm{C} / \mathrm{N}: 27$ y fósforo (disponible) $3.0 \mathrm{ppm}$. Antes de utilizar el suelo fue esterilizado con bromuro de metilo y posteriormente aireado durante 5 días.

Para cada especie se consideró un total de 48 plántulas, de las cuales 24 corresponden al tratamiento de inoculación, al que se adicionaron 100 $\mathrm{ml}$ de turba con inoculo de Glomus intraradices Schenck \& Smith (800 esporas +/- 50) y al control correspondiente se le aplicó un volumen equivalente de turba estéril. El ensayo de inoculación fue mantenido por un período de 20 semanas en in- 
vernadero, al término del cual se procedió a la fijación del material radical de 5 individuos (inoculados y control) por especie, obtenido al azar para su posterior tinción (Koske y Gemma, 1989) y cuantificación del grado de colonización micorrícica, de acuerdo a lo indicado por Giovannetti y Mosse (1980) y Godoy y Mayr (1989). Con el objeto de documentar la colonización por el simbionte en cada hospedero se realizaron observaciones al microscopio electrónico de barrido, según lo indicado por Godoy y Mayr (1989).

En forma paralela, las plántulas restantes, controles y tratamientos $(n=19)$ fueron cosechadas para medir las siguientes variables: diámetro de cuello de la raíz, longitud de tallo y raíz, peso fresco de tallo y raíz y peso seco de tallo y raíz. En adición, se estimó el índice de calidad de las plántulas de acuerdo a Ritchie (1984).

Los resultados fueron sometidos a un análisis de varianza 1-vía, y mediante el test de Tukey $a$ posteriori $(\mathrm{p}=0.5 \%)$ fue estimada la significancia estadística de los resultados.

\section{RESULTADOS}

En la tabla 1 se indican los valores medios de las diferentes variables cuantificadas, para las cuatro especies de coniferas inoculadas con Glomus intraradices y los controles respectivos.

En relación al diámetro del cuello de la raíz, se observa que en todas las especies los tratamientos con el inóculo micorrícico fueron superiores al control, con valores de significancia estadística a excepción de $F$, cupressoides (tabla 1). P. nubigena registró los máximos con 4.84 y $4.47 \mathrm{~mm}$, para las plántulas inoculadas y los controles, respectivamente. Con respecto a la variable largo del tallo, la totalidad de las especies inoculadas fueron significativamente superiores al control, cuyos máximos se obtuvieron en $S$. conspicua (tabla 1 , figura 1). Longitud de la raíz, variable que superó a la situación control en todos los tratamientos inoculados, no registró significancia estadística para la especie 5. conspicua, la cual se destaca, además, por poseer los mínimos valores en el crecimiento radical con 88.9 y $81.8 \mathrm{~mm}$, para plantas inoculadas y controles, respectivamente (tabla 1).

El peso fresco de tallo fue estimulado en todos los tratamientos con $G$. intraradices, cuyos valores en las especies estudiadas se diferenciaron estadísticamente de los controles correspondientes. Con respecto al peso fresco del sistema radical, se observa que fue notoriamente incrementado en los tratamientos con inoculación micorrícica, aunque los valores de $S$. conspicua con 4.08 y

Tabla 1

Valores medios (19 réplicas) de las diferentes variables en controles (-M) y tratamientos con Glomus intraradices $(+\mathrm{M})$ en especies de coniferas nativas del sur de Chile

Mean values (19 replications) of the different variables in controts (-M) and treatments wich Glomus intraradices (+M) in natives conifer species from Southern Chile

\begin{tabular}{|c|c|c|c|c|c|c|c|c|}
\hline \multicolumn{2}{|c|}{ Especie } & $\begin{array}{c}\text { DIAME } \\
(\mathrm{mm})\end{array}$ & $\begin{array}{l}\text { L. TALL } \\
(\mathrm{mm})\end{array}$ & $\begin{array}{c}\text { L. RAIZ } \\
(\mathrm{mm})\end{array}$ & $\begin{array}{c}\text { P.F.T. } \\
(\mathrm{g})\end{array}$ & $\begin{array}{l}\text { P.F.R. } \\
(\mathrm{g})\end{array}$ & $\begin{array}{c}\text { P.S.T. } \\
(\mathrm{g})\end{array}$ & $\begin{array}{l}\text { P.S.R. } \\
\text { (g) }\end{array}$ \\
\hline F.c & $(+\mathrm{M})$ & 3.95 & $252.6 *$ & $282.9 *$ & $5.60 *$ & $6.41 *$ & 1.78 & 1.02 \\
\hline F.c & $(-\mathrm{M})$ & 3.63 & 224.4 & 192.9 & 4.62 & 5.99 & 1.49 & 0.91 \\
\hline P.s & $(+\mathrm{M})$ & $4.24 *$ & $193.9 *$ & $135.8 *$ & $4.92 *$ & $3.84 *$ & 1.76 & 0.58 \\
\hline P.s & $(-M)$ & 3.45 & 156.5 & 110.5 & 3.60 & 2.32 & 1.29 & 0.55 \\
\hline S.c & $(+\mathrm{M})$ & $4.37 *$ & $297.9 *$ & 88.9 & $6.02 *$ & 4.08 & $2.17 *$ & 0.78 \\
\hline S.c & $(-M)$ & 3.61 & 260.3 & 81.8 & 4.90 & 3.75 & 1.76 & 0.58 \\
\hline P.n & $(+\mathrm{M})$ & $4.84 *$ & $191.6 *$ & $166.3 *$ & $5.54 *$ & $6.43 *$ & 2.16 & $0.95 *$ \\
\hline P.n & $(-M)$ & 4.47 & 170.0 & 121.3 & 4.23 & 5.63 & 1.85 & 0.77 \\
\hline
\end{tabular}

F.c. $=F$. cupressoides, P.s. $=P$. saligna, S.c. $=$ S. conspicua, P.n. $=P$. nubigena $(*$ significancia $\mathrm{p}=0.5 \%)$

DIAME= Diámetro cuello raíz, L. TALL= longitud tallo, L. RAIZ= longitud raíz, P.F.T.= peso fresco tallo, P.F.R.= peso fresco raíz, P.S.T.= peso seco tallo, P.S.R.= peso seco raíz. 


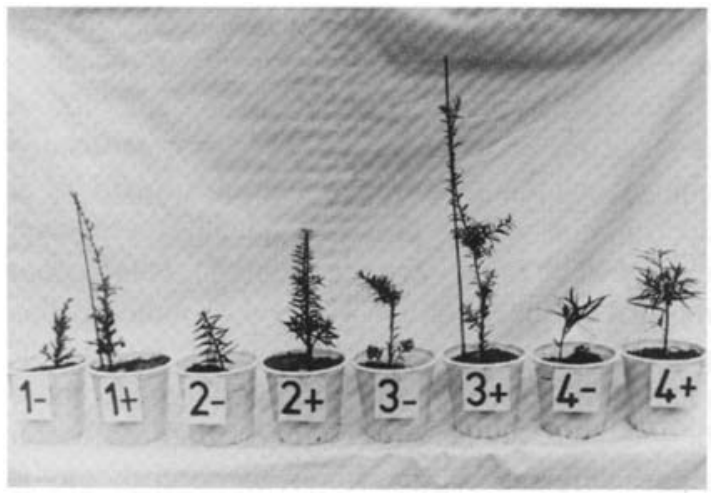

Figura 1. Bioensayo de inoculación de las coniferas nativas del sur de Chile con Glomus intraradices.

1. Fitzroya cupressoides

2. Podocarpus nubigena

3. Saxe-gothaea conspicua

4. Podocarpus saligna

-: Control y +: Tratamiento inoculado con micorriza.

Bioessay of inoculation with Glomus intraradices on native conifers from Southern Chile.

1. Fitzorya cupressoides

2. Podocarpus nubigena

3. Saxe-gothaea conspicua

4. Podocarpus saligna

-: Control and +: Treatment inoculated with mycorrhiza.

$3.75 \mathrm{~g}$ para las plántulas tratadas y controles, respectivamente, no presentaron diferencias significativas. El peso seco de tallo fue una variable que evidenció un aumento en todos los tratamientos versus controles respectivos, aunque valores con significancia estadística se presentaron únicamente en $S$. conspicua, con $2.17 \mathrm{~g}$ para plántulas inoculadas y $1.76 \mathrm{~g}$ en controles. Por último, el peso seco de raíz registra los mayores valores en aquellas plántulas inoculadas para todas las especies de coniferas investigadas, aunque con resultados significativos se observan exclusivamente en P. nubigena, con 0.95 y 0.77 g para plántulas tratadas y controles, respectivamente.

Al aplicar el índice de calidad de las plántulas, cuyos resultados se presentan en la tabla 2 , se observa que las especies de coniferas nativas investigadas registran valores estadísticamente significativos, respecto a los controles correspondientes. $P$. nubigena fue la especie con el mayor índice de calidad, tanto en las plántulas inoculadas (0.765) como para las plántulas controles (0.678). Aunque los valores del índice de calidad de $F$. cupressoides son relativamente mayores en las plantas tratadas con inoculantes, no se presentó significancia estadística respecto del control.
TABLA 2

Valores medios y desviación estándar del índice de calidad en plántulas de las especies de coniferas, inoculadas con Glomus intraradices $(+\mathrm{M})$ y $\operatorname{los}$ controles correspondientes (-M)

Mean values and standard deviation of the quality index in seedlings of the conifers species, inoculated with Glomus intraradices (+M) and control (-M), respectively

\begin{tabular}{|lll|}
\hline Especies & Media & \\
\hline F. cupressoides (+M) & 0.424 & $(+/-0.028)$ \\
F. cupressoides (-M) & 0.401 & $(+/-0.025)$ \\
& & \\
$P$. saligna $(+\mathrm{M})$ & $0.496^{*}$ & $(+/-0.037)$ \\
$P$. saligna $(-\mathrm{M})$ & 0.354 & $(+/-0.027)$ \\
S. conspicua $(+\mathrm{M})$ & $0.429^{*}$ & $(+/-0.020)$ \\
S. conspicua (-M) & 0.323 & $(+/-0.019)$ \\
$P$. nubigena $(+\mathrm{M})$ & $0.765^{*}$ & $(+/-0.044)$ \\
$P$. nubigena $(-\mathrm{M})$ & 0.678 & $(+/-0.042)$ \\
\hline
\end{tabular}

* significancia del tratamiento versus control $(\mathrm{p}=0.5 \%)$.

En las mediciones del grado de colonización micorrícica, en las coniferas, se observó que las variables analizadas presentaron un comportamiento específico para cada taxa analizado (tabla 3).

El mayor porcentaje de frecuencia e intensidad de micorrización correspondió a $S$. conspicua, con 78.6 y $23.7 \%$, respectivamente, mientras que $F$. cupressoides presentó los mínimos valores con 55.0 y $13.0 \%$, para frecuencia e intensidad, respectivamente. Las especies $P$. nubigena y $P$. saligna ocuparon una posición intermedia.

Un detalle de microfotografías de las raíces colonizadas por el endosimbionte $G$. intraradices es documentado en la fig. 2. Se observó en todas las especies de la familia Podocarpaceae la formación de nódulos radicales fuertemente colonizados por el hongo micorrícico vesículo-arbuscular, además de un extraordinario desarrollo de micelio extrarradical. Las raíces de $F$. cupressoides presentaron un gran desarrollo de arbúsculos, observables en diferentes estadios de desarrollo.

\section{DISCUSION}

El análisis de las variables morfométricas mostró diferencias entre las especies de plántulas inoculadas versus el control respectivo, destacándose 
TABLA 3

Frecuencia e intensidad del grado de colonización micorrícica en las especies de coníferas nativas del sur de Chile

Frequency and intensity of the mycorrhizal colonizaron degree in the native conifers species of Southern Chile

\begin{tabular}{|c|c|c|}
\hline Tratamientos & Frecuencia $(\%)$ & Intensidad $(\%)$ \\
\hline Fitzroya cupressoides & $55.0(+/-8.70)$ & $13.0(+/-5.50)$ \\
\hline Podocarpus nubigena & $63.3(+/-3.05)$ & $15.4(+/-5.24)$ \\
\hline Saxe-gothaea conspicua & $78.6(+/-4.60)$ & $23.7(+/-7.62)$ \\
\hline Podocarpus $\quad$ saligna & $59.4(+/-4.20)$ & $17.0(+/-5.10)$ \\
\hline
\end{tabular}

(\%): media y desviación estándar de tres paralelas.

como buenos parámetros indicadores aquellos derivados del tallo, particularmente longitud y peso fresco, correspondiendo a aquellos con una mayor frecuencia en la significancia estadística. Por el contrario, las variables peso seco de tallo y raíz constituyen los parámetros de menor frecuencia en su grado de significancia. Sin embargo, en términos de localización de la biomasa, F. cupressoides sería aquella especie que posee la mayor biomasa radical y cuya relación raíz/tallo fue de 0.57 , siendo de esta forma la que presenta la mejor relación, en comparación con sus congéneres, cuyos valores no sobrepasan el 0.5. Huante et al. (1993) indican que valores similares estarían indicando una adaptación a suelos con buen abastecimiento de nitrógeno y fósforo. Sin embargo, el efecto de la simbiosis micorrícicas en los patrones de localización de la materia seca, en ambientes con una disponibilidad de nutrientes contrastantes, es fuertemente variable (Allen, 1991).

En términos globales, todas las variables consideradas en las coniferas investigadas al término del bioensayo indicaron, para las plántulas inoculadas con $G$. intraradices, un efecto positivo del endosimbionte micorrícico.

Sin embargo, con el presente estudio se estima que el conjunto de variables consideradas constituyen un excelente indicador de la eficiencia del simbionte. En trabajos similares tendientes a cuantificar la eficiencia en los ensayos de inoculación micorrícica controlada, los parámetros utilizados son de uso frecuente (Furlan, et al., 1983; Bowen, 1987; Cuenca et al., 1990; Godoy et al., 1991; Huante et al., 1993).

En relación al índice de calidad, obtenido mediante el uso de los parámetros anteriormente señalados, se puede concluir que todos los tratamientos con inoculación micorrícica reflejan un efecto positivo del endosimbionte, aunque sólo tres especies presentaron diferencias significativas respecto al control, a saber: $P$. nubigena, $P$. saligna $y$. conspicua.

En relación al grado de micorrización observado en las especies de coniferas investigadas, se pudo constatar en general un óptimo grado de colonización al término del ensayo. Aunque los valores obtenidos no permiten diferenciar significativamente las especies, S. conspicua presentó los máximos valores. Las diferencias en frecuencia e intensidad obtenidos en las especies de coniferas dan cuenta de la especificidad que presenta cada uno de los hospederos bajo condiciones controladas, hecho documentado por Furlan et al. (1983), Cuenca et al. (1990), Godoy et al. (1991) y Huante et al. (1993).

En relación a la documentación fotográfica y morfoanatomía de la colonización de las raíces por el endosimbionte, se observó un patrón normal de colonización y estructura de las raíces y nódulos radicales, cuyos resultados son comparables a los obtenidos por Godoy y Mayr (1989).

Este hecho pone de manifiesto la necesidad de incrementar este tipo de estudios, tendientes a probar la compatibilidad y eficiencia de cepas nativas de hongos micorrícicos vesículo-arbusculares, con el objeto de optimizar procesos biotecnológicos que permitan, en un futuro próximo, implementar en forma masiva programas de inoculación en vivero y contribuir a la reforestación de áreas degradadas mediante la implementación de las técnicas de inoculación dentro de la tecnología silvícola existente en el país. Todo lo anterior permitirá proteger los recursos naturales renovables, en particular aquellas especies amenazadas o en vías de extinción, como es el caso particular de las coniferas nativas del sur de Chile. 

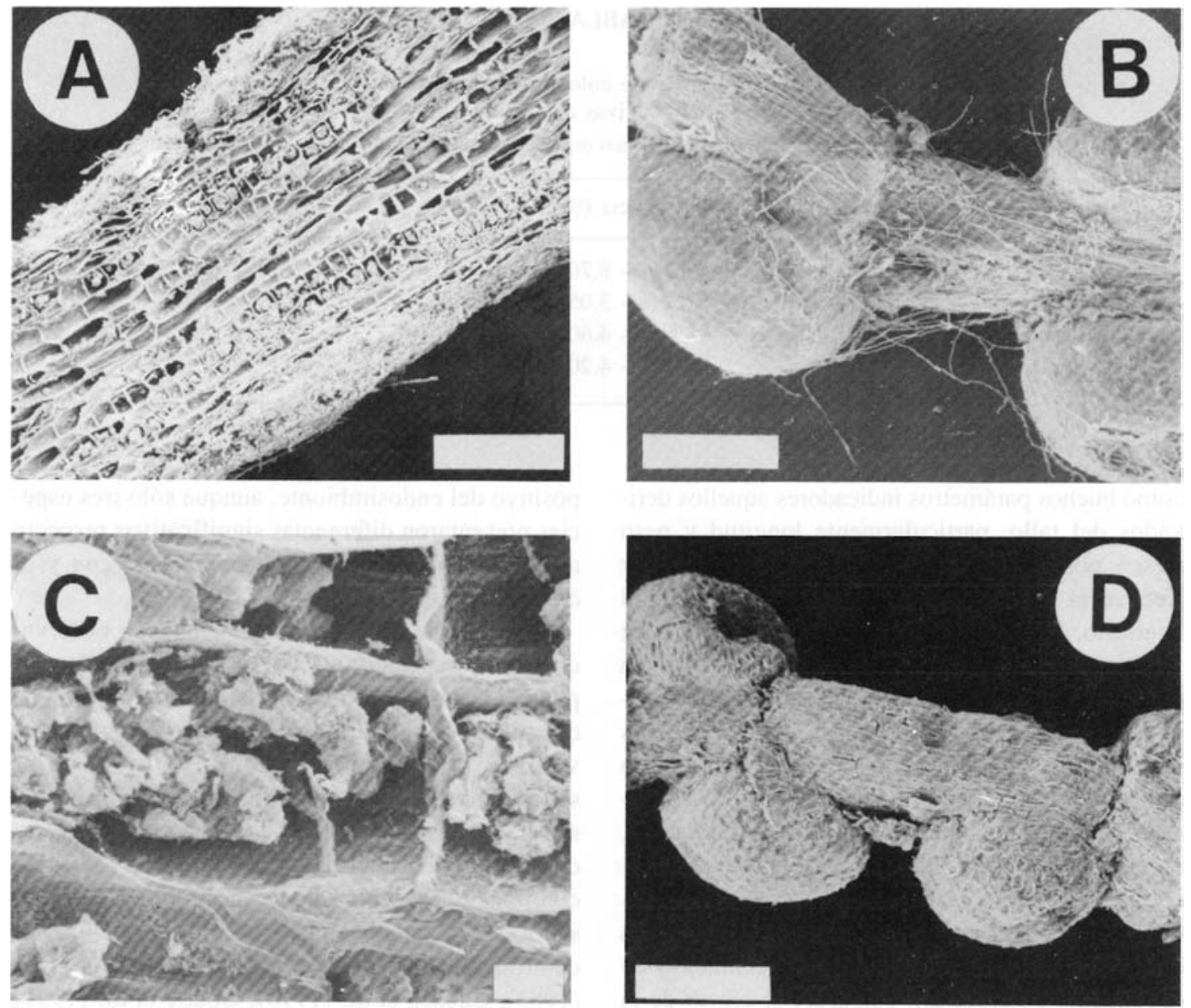

Figura 2. Microfotografías al microscopio electrónico de barrido de las raíces de plantas inoculadas con Glomus intraradices.

A= sección longitudinal de raíz en $F$. cupressoides, cuyas células están colonizadas por arbúsculos (escala $=0.5$ $\mathrm{mm})$.

$\mathrm{B}=$ nódulos en $P$. nubigena con micelio extrarradical (escala $1 \mathrm{~mm}$ ).

$\mathrm{C}=$ Detalle de arbúsculo en raíz de $S$. conspicua (escala 25 um).

$\mathrm{D}=$ Detalle de raíz con nodulo en $P$, saligna (escala $0.7 \mathrm{~mm}$ ).

Microphotos at Scanning Electron Microscopy of plant roots inoculated with Glomus intraradices.

$\mathrm{A}=$ Longitudinal section of root $\boldsymbol{F}$. cupressoides. showing cells with arbuscles (scale $=0.5 \mathrm{~mm}$ ).

$\mathrm{B}=$ Nodules in $\boldsymbol{P}$. nubigena and development of extramatrical hyphae $($ scale $=1 \mathrm{~mm})$.

$\mathrm{C}=$ Detail of arbuscles in root of 5 . conspicua (scale $=25 \mathrm{um}$ ).

$\mathrm{D}=$ Detail of nodulate root in $\boldsymbol{P}$. saligna $($ scale $=0.7 \mathrm{~mm})$.

\section{AGRADECIMIENTOS}

Los autores agradecen el apoyo brindado por la Corporación Nacional Forestal, DID-UACH S-94-07 y el Proyecto FONDECYT 0912-91.

Agradecemos la colaboración del Prof. Heriberto Figueroa en el análisis estadístico.

\section{BIBLIOGR AFIA}

ALLEN, M. 1991, The ecology of mycorrhizae. Cambridge University Press, Cambridge: $184 \mathrm{pp}$.

Allen, E., J. CHAMBERS, K. CONNOR, M. ALLEN y R. BROWN. 1989. "Natural reestablishment of Mycorrhizae in disturbed alpine ecosystems", Arctic and Alpine Research 19 (1): 11-20.

ARMESTO, J. 1992. "Mitos y realidades del bosque nativo chileno", Revista Chilena de Historia Natural 65(2): 173176.

ARMESTO. J., M. KALIN-ARROYO, P. LEON y C. SMITH. 
1992. "Problemas de conservación del bosque templado de Sudamérica", $4^{\circ}$ Encuentro científico sobre el Medio Ambiente. Ponencias, Tomo 1: 69-73.

BOWEN, G. 1987. The biology and physiology of infection and its development. En: Safir. G. (ed). Ecophysiology of VA mycorrhizal plants. C.R.C. Press, 223 pp.

CARRILlO, R., R. GODOY y H. PEREDO. 1992. "Simbiosis micorrícica en comunidades boscosas del valle central de Chile", Bosque 13 (2): 57-67.

CUENCA, G., R. HERRERA y E. MENESES. 1990. "Effects of VA mycorrhizal on the growth of cacao seedlings under nursery condition in Venezuela", Plant and Soil 126: 71 78.

DANIELSON, R. 1988. "Mycorrhizae in forestry. The state of the art in land reclamation". En: LONDE, M. y PICHE, Y. (eds.). Canadian Workshop on Mycorrhizae in Forestry: 39-41.

DONOSO, C. 1993. Bosques templados de Chile y Argentina. Variación, estructura y dinámica. Colección Nueva Técnica, Editorial Universitaria, $484 \mathrm{pp}$.

FURLAN, V., J. FORTIN y C. PLANCHETE. 1983. "Effects of differents vesicular-arbuscular mycorrhizal fungi on growth of Fraxinus americana", Can. Journal For. 13: 589593.

GIOVANNETTI, M. y B. MOSSE. 1980. "An evaluation of techniques for measuring vesicular" arbuscular mycorrhizal infections in roots"; New. Phytol. 84: 489-500.

GODOY, R. y R. MAYR. 1989. "Caracterización morfológica de mocorrizas VA en coniferas del Sur de Chile; Bosque 10(2): 89-98.

GODOY, R., C. RIQUELME, H. PEREDO y R. CARRILlO 1991. "Compatibilidad y eficiencia de hongos micorrícicos vesículo-arbusculares en Eucalyptus camaldulensis Dehnh. y Quillaja saponaria Mol", Ciencia e Investigación Forestal 5(2): 237-250.

GODOY, R., R. ROMERO, y R. CARRILlO. 1994. "Estatus micotrófico de la flora vascular en bosques de coniferas del sur de Chile", Revista Chilena ele Historia Natural 67(1): 209-220.

GRIMME, J., J. MACKEY, S. HILLIER y D. READ. 1989 "Floristic diversity in a model system using experimental microcosm", Nature 328: 420-422.
HARLEY, J. 1989. "The significance of mycorrhiza", Micol. Res. 92(6): 641-655.

HARTMANN, H. y D. KESTER. 1964. Propagación de plantas. Principios y prácticas. Edit. C.E.C.S.A., México, 814 pp.

HILDEBRAND-VOGEL, R., R. GODOY, A. VOGEL y R. CARRILLO. 1992. "Fitzroya cupressoides (Mol) Johnst. primary forest of southern Chile. Flora, vegetation and mycorrhiza". En: Song YONGCHANG (ed.). Proceedings of the 35th Symposium of IAVS in Shangai-China (en prensa).

HUANTE, P., E. RINCON y E. ALLEN. 1993. "Effect of vesicular-arbuscular mycorrhizae on seedling growth of four tree species from the tropical deciduous forest in México", Mycorrhiza 2:141-145.

KOSKE R. y J. GEMMA. 1989. "A modified procedure for staining roots to detect VA mycorrhizae", Mycol. Res. 92(4): 486-488.

LARA, A. y T. VEBLEN. 1993. "Forest plantations in Chile: a successfull model". En: MATHER, A. (edit). Afforestation policies, planning and progress. Belhaben Press, LondonFlorida, pp. 118-139.

Mc NEILL, L. 1989. "Estrategias para un desarrollo económico viable", Investigación y Ciencia 158: 115-124.

PARKER, T. y C. DONOSO. 1993. "Natural regeneration of Fitzroya cupressoides in Chile and Argentina", Forest Ecology and Management 59:63-85.

PERRY, D., M. MOLINA y M. AMARANTHUS. 1987. "Mycorrhizae, mycorrhizospheres and reforestation: current knowledge and research needs". Can. Journal Forestry Research 17: 929-940.

READ, D. 1991. "The role of the mycorrhizal simbiosis in the nutrition of plant communities". En: Ecophysiology of ectomycorrhizae offorest trees. The Marcus Wallenberg Foundation, Symposia Proceeding 7: 27-53.

RITCHIE, G. 1984. "Assessing seedling quality". En: DURYEA, M \& LANDIS, T. (eds.). Forest Nursery Manual: production ofbareroot seedlings. The Hague, Martinus Nijhoff, Dr. Junk: 243-259. 